\title{
Thermo-sensitive Electrospun Fibrous Magnetic Composite Sheets
}

\author{
Jungsu Choi ${ }^{1}$, Jinu Kim ${ }^{2}$, Heejae Yang ${ }^{1}$, Frank K. Ko ${ }^{1}$, and Ki Hyeon Kim ${ }^{2 *}$ \\ ${ }^{1}$ Department of Materials Engineering, University of British Columbia, Vancouver, V6T 1Z4, Canada \\ ${ }^{2}$ Department of Physics, Yeungnam University, Gyeongsan 38541, Korea
}

(Received 11 August 2015, Received in final form 25 August 2015, Accepted 29 August 2015)

\begin{abstract}
The PVDF fibrous composite filled with iron oxide nanoparticles were prepared by using the electrospinning technique. The electrospun composite have the thickness in the range of $60-80 \mu \mathrm{m}$ with the average fibrous diameters of 500-900 nm. The magnetizations of PVDF fibrous composite filled with iron oxide nanoparticles showed $4.5 \mathrm{emu} / \mathrm{g}, 3.1 \mathrm{emu} / \mathrm{g}$ and $1.6 \mathrm{emu} / \mathrm{g}$ at $1.5 \mathrm{~T}$ of external magnetic field for $20 \mathrm{wt} \%, 10 \mathrm{wt} \%$ and 5 wt.\% iron oxide nanoparticles, respectively. The heat elevation of the magnetic composite were measured under various AC magnetic fields, frequency and the ambient temperatures. The temperature reached up to $46.3^{\circ} \mathrm{C}$ from $36^{\circ} \mathrm{C}$ at 128 Oe and $355 \mathrm{kHz}$ for $20 \mathrm{wt}$.\% iron oxide nanoparticles filled in PVDF fibrous composite sheet. The specific absorption rate of theses sheets increased from $0.041 \mathrm{~W} / \mathrm{g}$ to $0.236 \mathrm{~W} / \mathrm{g}$ with the increment of $\mathrm{AC}$ magnetic field from 90 Oe to 167 Oe at $190 \mathrm{kHz}$, respectively.
\end{abstract}

Keywords : iron oxide, alternative magnetic field, specific absorption ratio, fiber

\section{Introduction}

MAGNETIC NANOPARTICLES (MNPs) have a high potential for bio-applications such as magnetic resonance imaging contrast enhancement, tissue repair, drug delivery and hyperthermia applications [1-4]. Among the various MNPs, magnetite $\left(\mathrm{Fe}_{3} \mathrm{O}_{4}\right)$ is one of the most important spinel-type particles with superparamagnetic property. It has been known that the important properties of $\mathrm{Fe}_{3} \mathrm{O}_{4}$ MNPs for biomedical applications are nontoxicity, biocompatibility, low particle dimension, large surface area, and suitable magnetic properties [4]. These magnetic materials can be tailored with the various shapes such as particles, rod, wire, and their composites to improve the magnetic properties and biocompatibility [5-8]. In general, the magnetic thermo-sensitive materials especially, MNPs and their colloids have been widely studied in local hyperthermia [1-8]. To apply the hyperthermia of MNPs, it should evenly disperse throughout the fluid. And it must be shorten treatment time with the maximized loss. The magnetic hyperthermia provides the heat at the site of the tumor invasively by applying an external alternating magnetic field (AMF) to the MNPs at the tumor site.the

CThe Korean Magnetics Society. All rights reserved.

*Corresponding author: Tel: +82-53-810-2334

Fax: +82-53-810-4616, e-mail: kee1@ynu.ac.kr heat range about $42^{\circ} \mathrm{C}$ to $46^{\circ} \mathrm{C}$ for cancer therapy [1]. The magnetic field-induced heating of MNPs occurs through a combination of eddy currents, hysteresis losses, and relaxation losses. The eddy current loss is deeply related with the skin depth of materials which is defined by the applied frequency, conductivity and permeability of materials. In multidomain magnetic particles, the loss is mainly governed by the hysteresis loss although Neel relaxation is generated by magnetic spins in singledomain MNPs. These magnetic domain depends on the size of MNPs. In the Brownian relaxation, the interaction between a thermal force and viscous drag in the suspending medium will influence the particle movement significantly. The losses of the MNPs is governed by the intrinsic magnetic properties as well as the strength of the AMF and their operating frequencies [4]. These magnetic thermal losses can be expressed by Specific Absorption Rate (SAR). In recent, researchers have been tried to apply the composite sheet instead of magnetic colloids to healing of skin infections including hyperthermia-aided immunotherapy [9-14]. The magnetic fibrous composite (MFC) sheet has an advantage of easily usage to healing and wound target area with an immovable MNPs. And it can be expected to remain locally keeping the MNPs in the surroundings of the tumor, thus allowing a repeated localized heating. The MFC are made through electrospinning which process allows the evenly distribution of 
MNPs in composites and design of polymeric fibers with a good flexibility. Electrospinning is well known as an efficient tool to fabricate multifunctional fibrous matrices, which are composed of polymeric fibers with diameters ranging from hundreds of nanometers to several micrometers [15]. Therefore, to verify the heat behaviors of MFC, we synthesized the PVDF fibrous composite filled with iron oxide nanoparticles (IONPs) by using electrospinning technique.

\section{Experimental Procedure}

The fibrous composite sheets filled with IONPs were fabricated using the electrospinning technique as shown in Fig. 1(a). For electrospinning, the spinning dopes were prepared by sonication of the IONPs $\left(98 \% \mathrm{Fe}_{3} \mathrm{O}_{4}\right.$, SkySpring Nanomaterials, 10-20 nm) in Dimethylformamide (DMF, Sigma-Aldrich) for 24 hours. $20 \mathrm{wt} \%$ of Polyvinylidene Fluoride (PVDF, Kynar) to DMF were then added to the prepared IONPs/DMF mixture. The solutions mixtures were magnetically stirred for 24 hours before the electrospinning at $90^{\circ} \mathrm{C} .5,10$ and $20 \mathrm{wt} . \%$ of $\mathrm{Fe}_{3} \mathrm{O}_{4}$ to PVDF were prepared for comparison. The prepared solutions were electrospun using NEU (Kato Tech) electrospinning system as shown Fig. 1(b). $5 \mathrm{ml}$ of each spinning dope was transferred to a syringe for electrospinning. The distance between spinneret to target was set as $15 \mathrm{~cm}$ and gauge 18 blunt ended syringe needle was used as spinneret. The voltage applied to the spinning nozzle was varied between $10-15 \mathrm{kV}$ to obtain stable and continuous fiber fabrication while solution was pumped at a rate of $0.1 \mathrm{ml} / \mathrm{min}$.

Figure 1(c) shows the typical flexible PVDF fibrous composite sheet filled with IONPs. The morphologies and structure of composite sheets were determined by using Field Emission Scanning Electron Microscopy (FE-SEM, Philips s-4800) and X-ray diffractometer (XRD, $\mathrm{Cu} \mathrm{K} \alpha, \lambda$ $=1.54059 \AA)$. The magnetic properties of the particles were measured by using Vibrating Sample Magnetometer (VSM, Lakeshore 7410). The induction heat temperature were measured by AC heating system which is composed of RF power supply (AMERITHERM INC., HOTSHOT $2.4 \mathrm{~kW}$ ) and 5.5 turns-helical shaped $\mathrm{Cu}$ coil with the 80 $\mathrm{mm}$-height and $70 \mathrm{~mm}$-inner diameter as an $\mathrm{AC}$ magnetic field applicator. The temperature and the intensity of AMF was measured by optical thermal sensors and 4 channel thermometer (FISO TMI4) with a magnetic field transducer (SENIS AG.), respectively.

\section{Results and Discussion}

Figure 2 shows the optical images of the PVDF fibrous composite sheets filled with IONPs and FESEM images of their surfaces and cross sectional views with the increment of IONPs inclusion from 0 wt.\%, 5 wt.\%, 10 wt. $\%$ and $20 \mathrm{wt} . \%$, respectively. The average thickness of composites is about $70 \mu \mathrm{m}$ and the fiber diameter of about 500-900 nm. Any aggregation of fibers and external particles on surface of fibers were not existed. The XRD peaks of the synthesized PVDF fibrous composite filled with IONPs were compared with those of the iron oxide powder and PVDF fibrous sheet without inclusion of IONPs as shown in Fig. 3. The diffraction pattern of iron oxide powder exhibited the partially mixed magnetic phases with $\alpha-\mathrm{Fe}_{2} \mathrm{O}_{3}, \alpha-\mathrm{Fe}$ and etc., which a strong diffraction peak corresponds to (220), (311), (400), (422), (511) and (440) reflections of $\mathrm{Fe}_{3} \mathrm{O}_{4}$ and (104), (110) reflections of hematite $\left(\alpha-\mathrm{Fe}_{2} \mathrm{O}_{3}\right)$, respectively. (a)

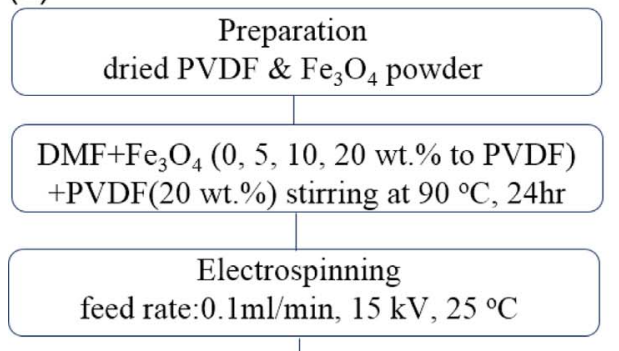

\begin{tabular}{c} 
Drying fiber \\
$2 \mathrm{hr}, 105^{\circ} \mathrm{C}$ in vacuum \\
\hline $\begin{array}{c}\text { Fiber post-processing } \\
170^{\circ} \mathrm{C}, 2 \mathrm{hr} \text { at Ar atmosphere }\end{array}$
\end{tabular}

(b)

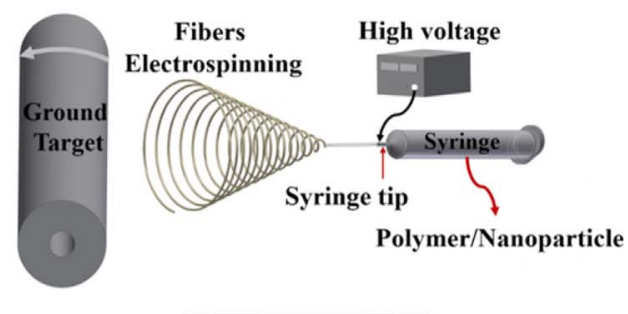

(c)

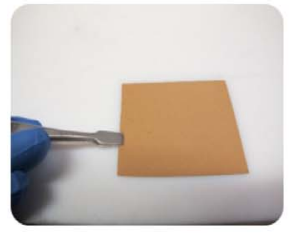

Fig. 1. (Color online) Synthesis process (a), schematic of electrospinning system (b) and optical image (c) of the fabricated PVDF fibrous composite sheet filled with IONPs, respectively. 


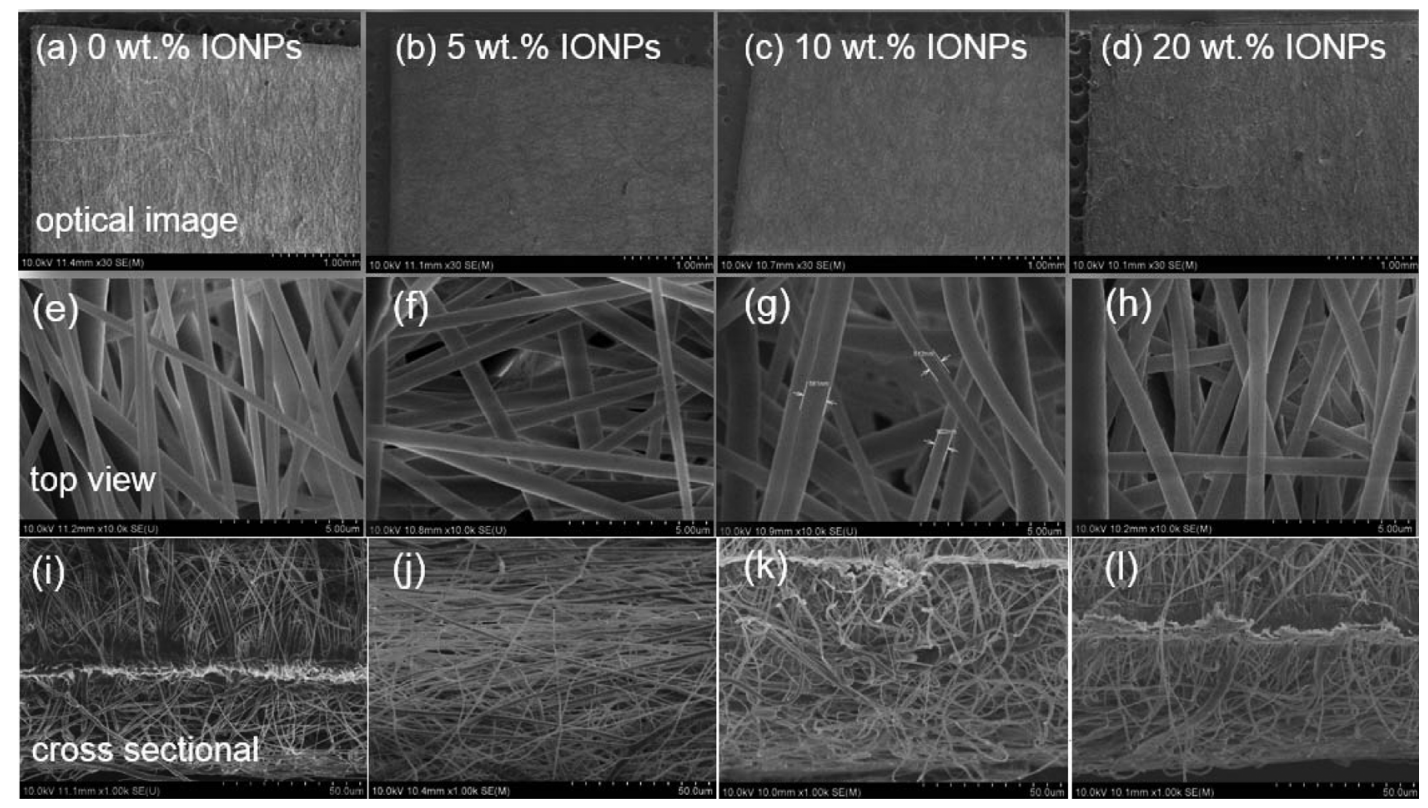

Fig. 2. Optical (a)-(d) and FESEM (e)-(l) images for sheets surface and cross sectional view of the fibrous PVDF composite sheets filled with the change of IONPs contents; 0 wt.\%, 5 wt.\%, $10 \mathrm{wt} . \%$, and $20 \mathrm{wt} . \%$, respectively.

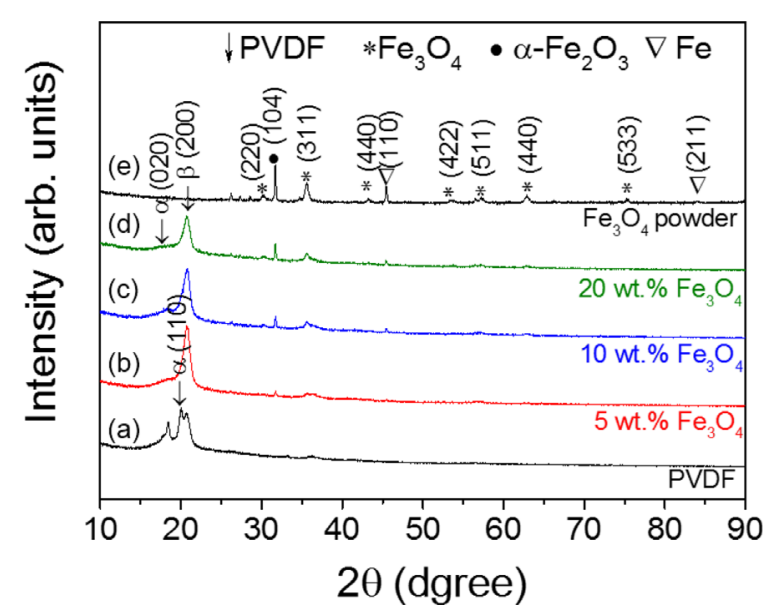

Fig. 3. (Color online) X-ray diffraction patterns of PVDF fibrous mat without IONPs (a) and IONPs contents of 5 wt.\% (b), 10 wt.\% (c), 20 wt.\% (d) and IONPs powders (e), respectively.

The magnetization value of iron oxide powder without polymer exhibited about $35.5 \mathrm{emu} / \mathrm{g}$ at room temperature, which the external magnetic field was applied up to $1.5 \mathrm{~T}$ as shown in Fig. 4 (inset). The magnetizations of PVDF fibrous composites filled with IONPs of $5 \mathrm{wt} . \%, 10 \mathrm{wt} . \%$ and $20 \mathrm{wt} . \%$ were obtained $1.6 \mathrm{emu} / \mathrm{g}, 3.1 \mathrm{emu} / \mathrm{g}$ and 4.5 emu/g, respectively in Fig. 4. The magnetic squareness $\left(\mathrm{M}_{\mathrm{r}} / \mathrm{M}_{\mathrm{s}}\right)$ of PVDF fibrous composites with IONPs are very small values (below 0.09) for all samples. It indicates that the 10-20 nm sized IONPs in PVDF fibrous

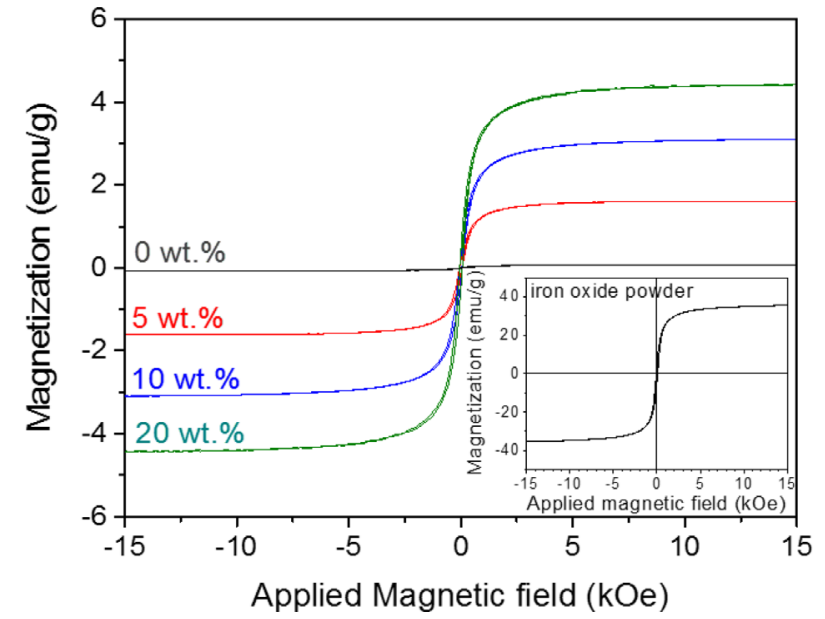

Fig. 4. (Color online) The magnetization curves at $300 \mathrm{~K}$ with the increment of IONPs contents in PVDF fibrous composite sheet from 0 wt. $\%$ to $20 \mathrm{wt} . \%$ in comparison with that of IONPs without polymerization process (Inset), respectively.

composites could be superparamagnetic particles. The heat contributions of these particles in the range of $\mathrm{kHz}$ frequency, the eddy current and hysteresis loss can be negligible due to the large skin depth and very low coercivity.

To verify the heat loss of the PVDF fibrous composites filled with IONPs, the specimens were placed in an isolated air environment. The ambient temperature was kept constant with $10^{\circ} \mathrm{C}, 20^{\circ} \mathrm{C}$ and $36^{\circ} \mathrm{C}$ in a temperature controllable chamber, respectively. The intensity of AMF 


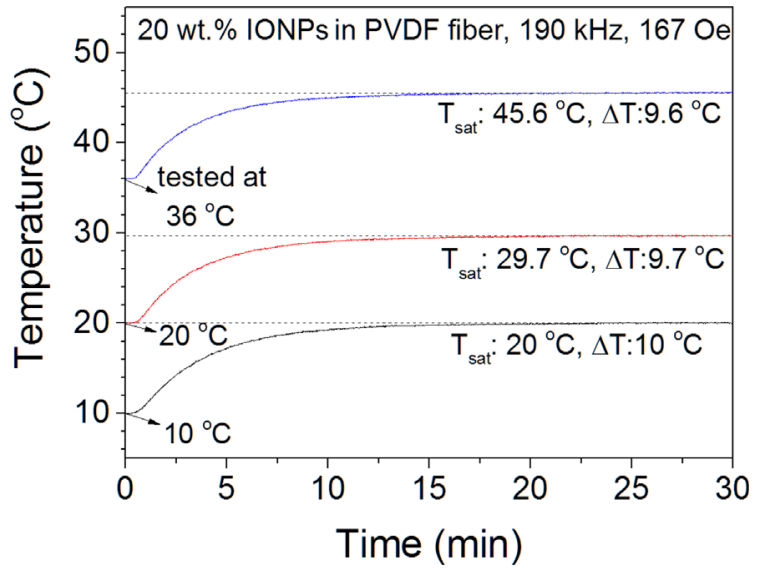

Fig. 5. (Color online) Temperature increment as a function of time for PVDF fibrous composite sheets filled with IONPs of $20 \mathrm{wt} . \%$ at the change of ambient temperatures of 10, 20, and $36^{\circ} \mathrm{C}$ under $190 \mathrm{kHz}$ and $167 \mathrm{Oe}$, respectively.

was generated by using a 5.5 turn coil with 90 Oe, 128 Oe and $167 \mathrm{Oe}$ at the selective frequency of $190 \mathrm{kHz}, 250$ $\mathrm{kHz}$ and $355 \mathrm{kHz}$, respectively. When these PVDF fibrous composites filled with IONPs exposed to alternating magnetic field, they were heated by relaxation loss. To compare the heat behaviors with the different ambient temperatures, the heat behaviors of PVDF fibrous com-
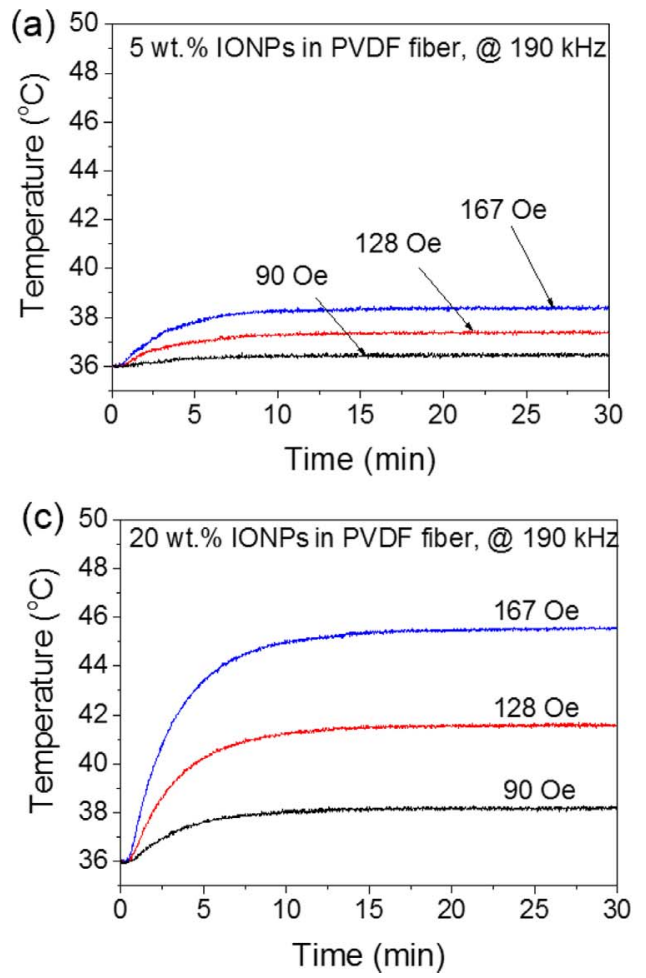

posites with 20 wt.\% IONPs were measured with the different ambient temperatures at $10^{\circ} \mathrm{C}, 20^{\circ} \mathrm{C}$ and $36^{\circ} \mathrm{C}$ for, respectively, as shown in Fig. 5. As results, the increasing temperatures $(\Delta \mathrm{T})$ stably exhibited about $10^{\circ} \mathrm{C}$ regardless of the different initial temperatures. Therefore, we selected the initial ambient temperature of $36^{\circ} \mathrm{C}$ which is similar with that of human body. Figure 6 shows the comparison of the elevated temperature for the PVDF fibrous composites field with 5 wt.\%, 10 wt. $\%$ and 20 wt.\% IONPs with the change of applied AMF intensities from $90 \mathrm{Oe}, 128 \mathrm{Oe}$ and $167 \mathrm{Oe}$ at the fixed frequency of $190 \mathrm{kHz}$, respectively. The temperatures were linearly increased with the increment of inclusions of IONPs and intensity of AMF from 90 Oe to 167 Oe. Figure 6(d) shows the increasing temperature $(\Delta \mathrm{T})$ with the increment of IONPs and intensity of AMF. The maximum $\Delta \mathrm{T}$ exhibited about $9.6^{\circ} \mathrm{C}$ for $20 \mathrm{wt} . \%$ IONPs at 167 Oe and $190 \mathrm{kHz}$. To evaluate the frequency dependence of heat, the elevated temperatures of PVDF fibrous composites with IONPs were measured at $90 \mathrm{kHz}, 250 \mathrm{kHz}$ and 355 $\mathrm{kHz}$ with the fixed AMF of $128 \mathrm{Oe}$, respectively, as shown in Fig. 7. The temperatures were increased with the increment of frequency and inclusion of IONPs contents, respectively. The $\Delta \mathrm{T}$ increased up to $10.3^{\circ} \mathrm{C}$ with the increment of frequency and inclusion of IONPs,
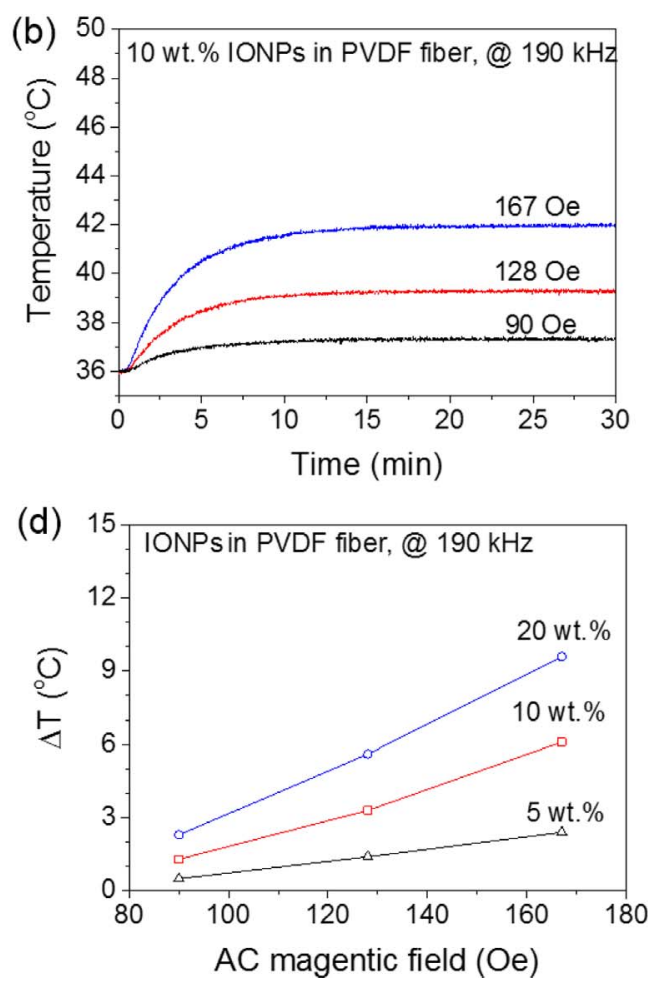

Fig. 6. (Color online) Temperature as a function of time for the PVDF fibrous composite sheets with the change of IONPs contents; 5 wt.\% (a), 10 wt.\% (b), and 20 wt.\% (c), the elevated temperature differences $(\Delta \mathrm{T})(\mathrm{d})$ for the change of AMF 90 Oe, 128 Oe and $167 \mathrm{Oe}$ at $190 \mathrm{kHz}$, respectively. 

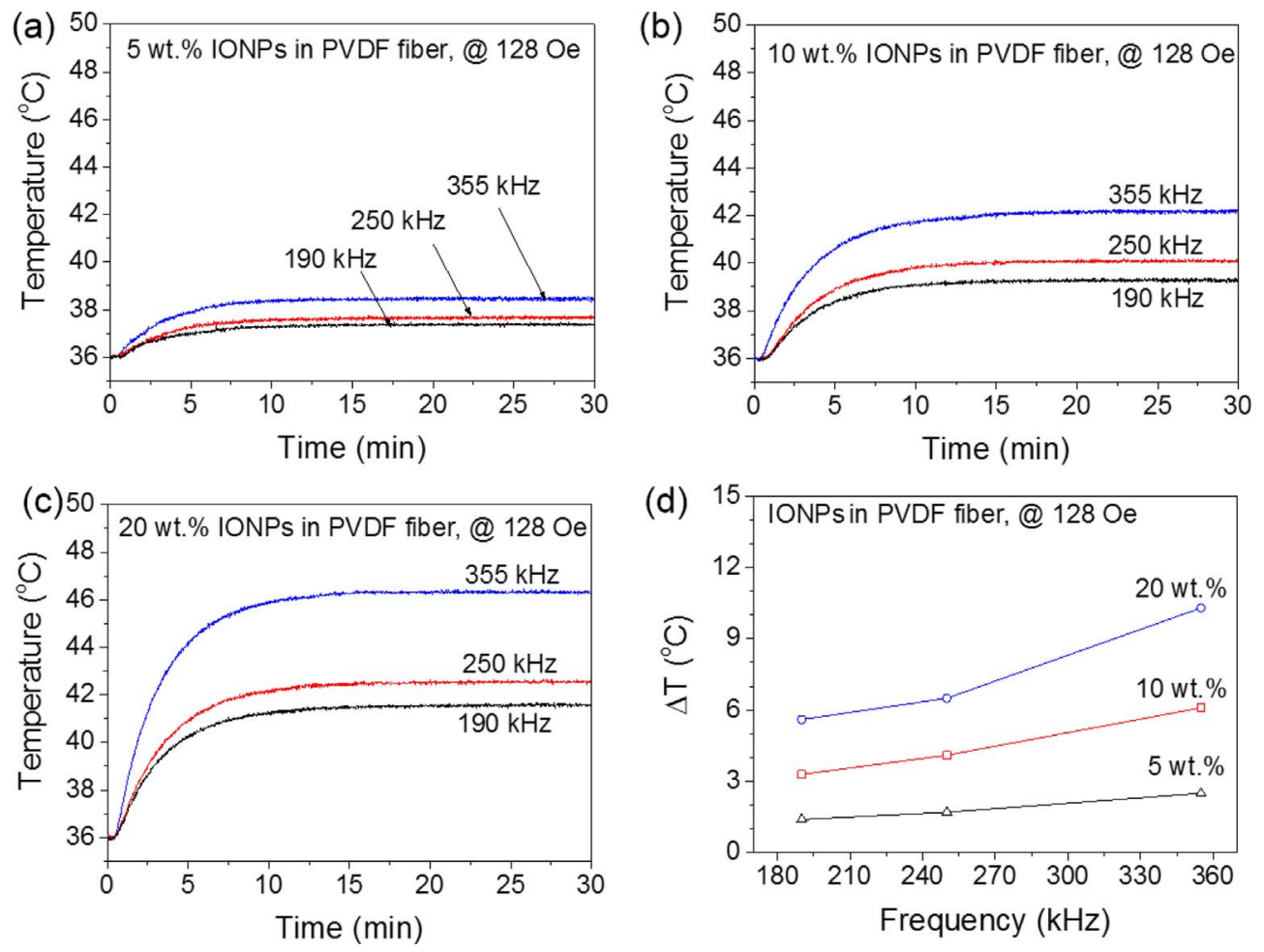

Fig. 7. (Color online) Temperature as a function of time for the PVDF fibrous composite sheets with the change of IONPs contents; 5 wt.\% (a), 10 wt.\% (b), and 20 wt.\% (c), the elevated temperature differences $(\Delta \mathrm{T})(\mathrm{d})$ for the change of frequency from 190 $\mathrm{kHz}$ to $355 \mathrm{kHz}$ at $128 \mathrm{Oe}$, respectively.

as shown in Fig. 7(d). These magnetic losses can be expressed by the specific loss power (SLP) (also called the specific absorption rate (SAR)), which is defined as the thermal dissipation per unit of mass of the magnetic material in the presence of an AMF. SLP is equal to $\mathrm{c} \times$

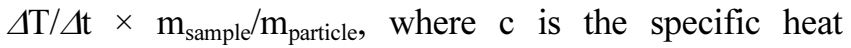
capacity of sample, $m_{\text {sample }}$ is the mass of sample, $m_{\text {particle }}$ is the mass of the magnetic material in specimen, and $\Delta \mathrm{T} /$ $\Delta \mathrm{t}$ is the slope of the heating curve. For nanoparticle in composites, the heat capacity of the system was corre-

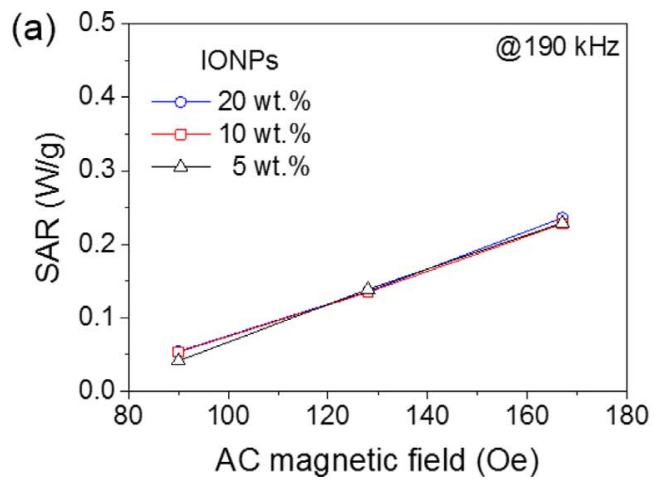

spond to $\left(\mathrm{m}_{\text {particle }} \times \mathrm{c}_{\text {particle }}+\mathrm{m}_{\text {composite }} \times \mathrm{c}_{\text {composite }}+\mathrm{m}_{\text {air }} \times\right.$ $\left.c_{\text {air }}\right) /\left(m_{\text {particle }}+m_{\text {composite }}\right)$, where $m$ is the mass of magnetic particles $[4,16]$.

Figure 8 shows the calculated the SAR based on measured the temperature increasing ratio $(\Delta \mathrm{T} / \Delta \mathrm{t})$ with the change of $\mathrm{AMF}$, frequency and the increment of IONPs inclusion in PVDF fibrous composite. The SAR values were not so changed with the IONPs contents in fibers. However, they increased up to $0.24 \mathrm{~W} / \mathrm{g}$ with the increment of $\mathrm{AMF}$ and $0.33 \mathrm{~W} / \mathrm{g}$ with the increment of

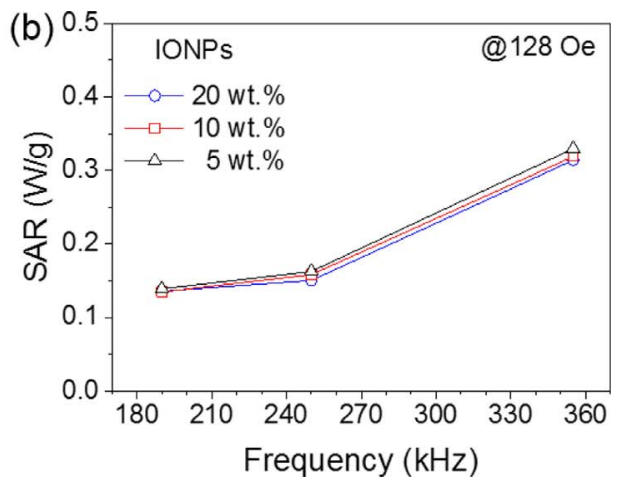

Fig. 8. (Color online) SAR with the change of magnetic fields at $190 \mathrm{kHz}$ (a) and frequency at 128 Oe (b) for the change of IONPS contents of $5 \mathrm{wt} . \%, 10 \mathrm{wt} . \%$, and $20 \mathrm{wt} . \%$, respectively. 
frequency, respectively.

\section{Conclusion}

The heating effects of PVDF fibrous composite filled with IONPs were studied in various strengths of AMF and frequencies. All the magnetic fibrous composite showed that the temperatures increased with the increment of AMF, frequency and IONPs inclusion, respectively. The increasing heat temperatures $(\Delta \mathrm{T})$ exhibited almost same values even though the initial ambient temperatures were different with $10^{\circ} \mathrm{C}, 20^{\circ} \mathrm{C}$ and $36^{\circ} \mathrm{C}$. The maximum heat temperature and SAR showed $46.3^{\circ} \mathrm{C}$ and $0.33 \mathrm{~W} / \mathrm{g}$ for the 20 wt.\% IONPs at $128 \mathrm{Oe}, 355 \mathrm{kHz}$ respectively. SAR values may not dominant on IONPs contents in fibers but the strength of AMF and their frequency. As results, these flexible PVDF fibrous composite sheet filled with IONPs could be helpful for hyperthermia treatment and skin wound healing applications.

\section{Acknowledgement}

This work was supported by the 2014 Yeungnam University Research Grant.

\section{References}

[1] D.-H. Kim, D. E. Nikles, D. T. Johnson, and C. S. Brazel, J. Magn. Magn. Mater. 320, 2390 (2008).

[2] S. Mornet, S. Vasseur, F. Grasset, P. Veverka, G. Goglio,
A. Demourgues, J. Portier, E. Pollert, and E. Duguet, Prog. Solid State Chem. 34, 237 (2006).

[3] C. C. Berry and A. S. G. Curtis, J. Phys. D: Appl. Phys. 36, R198 (2003).

[4] I. Baker, Q. Zeng, W. Li, and C. R. Sullivan, J. Appl. Phys. 99, $08 \mathrm{H} 106$ (2006).

[5] C. Sun, J. S. H. Lee, and M. Zhang, Advanced Drug Delivery Reviews 60, 1252 (2008).

[6] A. K. Gupta and M. Gupta, Biomaterials 26, 3995 (2005).

[7] D. Caruntu, G. Caruntu, and C. J. O’Conner, J. Phys. D 40, 5801 (2007).

[8] M. Thankur, K. De, S. Giri, S. Si, A. Kotal, and R. K. Mandal, J. Phys.:Condens. Matter. 18, 9093 (2005).

[9] T.-C. Lin, F.-H. Lin, and J.-C. Lin, Acta Biomater. 8, 2704 (2012).

[10] J. Kim, S. Zhu, Y. X. Gan, and K. Forward, Adv. Res. 3, 84 (2015).

[11] C. Huang, S. J. Soenen, J. Rejman, J. Trekker, L. Chengxun, L. Lagae, W. Ceelen, C. Wilhelm, J. Demeester, and S. C. De Smedt, Adv. Funct. Mater. 22, 2479 (2012).

[12] H. Wu, R. Zhang, X. Liu, D. Lin, and W. Pan, Chem. Mater. 3, 3506 (2007).

[13] M. Miyauchi, T. J. Simmons, J. Miao, J. E. Gagner, Z. H. Shriver, U. Aich, J. S. Dordick, and R. J. Linhardt, ACS Appl. Mater. Interfaces 19, 1958 (2011).

[14] C.-H. Park, S.-J. Kang, L. Tijing, H. R. Pant, and C. S. Kim, Ceram. Int. 39, 9785 (2013).

[15] N. Bhardwaj and S. C. Kundu, Biotechnol. Adv. 28, 325 (2010).

[16] Andrzej Skumiel, J. Magn. Magn. Mater. 307, 85 (2006). 